\title{
Original article \\ The use of sublingual fentanyl for breakthrough pain by using doses proportional to opioid basal regimen
}

\section{Sebastiano Mercadante}

Giovanna Prestia

Anesthesia and Intensive Care Unit \& Pain Relief and Palliative Care Unit, La Maddalena Cancer Center, Palermo, Italy

\section{Alessandra Casuccio \\ Chair of Clinical Neuroscience, University of Palermo, Palermo, Italy}

\section{Address for correspondence:}

Sebastiano Mercadante MD, Director, Anesthesia \& Intensive Care Unit and Pain Relief \& Palliative Care Unit, La Maddalena Cancer Center, Via S. Lorenzo 312, 90145 Palermo, Italy.

Tel.: +39091 6806521; Fax +390916806110; terapiadeldolore@lamaddalenanet.it

\section{Keywords:}

Breakthrough-episodic pain - Cancer pain - Opioids Sublingual tablet

Accepted: 12 July 2013; published online: 19 August 2013 Citation: Curr Med Res Opin 2013; 1-6

\section{Abstract}

\section{Objective:}

The aim of this study was to prospectively assess the efficacy and safety of sublingual fentanyl (SLF) in doses proportional to opioid doses used for background analgesia for the treatment of BTP of cancer patients.

\section{Methods:}

A sample of patients admitted to an acute palliative care unit, presenting breakthrough pain (BTP) episodes and receiving stable doses of opioids for background pain was selected to assess the efficacy and safety of SLF used in doses proportional to the basal opioid regimen used for the management of BTP. For each patient, data from four consecutive episodes were collected. For each episode, nurses collected changes in pain intensity and adverse effects when pain got severe (T0), and 5, 10, and 15 minutes after SLF was given (T15).

\section{Results:}

Seventy patients were recruited for the study. The mean age was $61.7( \pm 11.5)$. Forty-one patients were males. A total of 173 episodes of BTP were recorded (mean 2.5 episodes/patient). In 19 events, documentation regarding changes in pain intensity was incomplete. Of the 154 evaluable episodes, 143 were successfully treated (92\%). Mean doses of SLF were $637 \mu \mathrm{g}$ (SD 786), and 51 patients $(72.8 \%)$ received SLF doses $\geq 800 \mu \mathrm{g}$. When compared to younger adult patients, older patients received significantly lower doses of FBT $(p<0.0005)$, similarly to their lower basal opioid regimen. Pain intensity significantly decreased at T5, 10 and T15 $(p<0.0005)$. The number of patients with a pain reduction of more than $33 \%$ at $T 5, T 10$, and $T 15$ were 11,79 , and 137 , respectively, and the number of patients with a reduction in pain intensity of more than $50 \%$ were $1,21,114$ at the same intervals, respectively. № differences in changes in pain intensity for gender $(p<0.9)$ or age $(p<0.85)$ were observed. No significant changes in the number of patients reporting adverse effects of mild-moderate intensity were reported after SLF administration in comparison with baseline, and no adverse effects severe enough in intensity to require medical intervention were observed. Limitations of this study are represented by the uncontrolled design.

\section{Conclusion:}

This study suggests that SLF given in doses proportional to the basal opioid regimen for the management of BTP is safe and effective in clinical practice.

\section{Introduction}

According to a prevalent definition, breakthrough pain (BTP) is a transitory exacerbation of pain, severe in intensity and with a rapid onset, superimposed on an otherwise stable pain pattern in patients treated with opioids ${ }^{1-3}$. The presence of BTP has been considered as a negative prognostic factor, and influences the quality of life of these patients ${ }^{4}$. The availability of supplemental doses of 
opioids in addition to continuous analgesic medication is the main treatment suggested for management of these pain flares, either during dose titration or when basal pain is under control. Anecdotal experience with oral opioids suggests that an effective dose of BTP medication must be a percentage of a patient's total daily opioid dose $^{5}$. However, the onset time of oral opioids is expected to be 30-45 minutes. As pain relief is usually required urgently in most cases, in the last decade new routes of administration have been designed to provide fast pain relief. The use of these fentanyl delivery systems, commonly named rapid onset opioids (ROOs) has been shown to provide the best effective treatment in comparison with placebo or oral morphine ${ }^{6}$.

Most trials performed with ROOs suggest titrating doses to achieve an effective dose, as there is no relationship between effective fentanyl dose and a fixed schedule opioid regimen $^{7-9}$. However, the evidence is only indirect, because it was never the primary outcome and was derived from the study design rather than on convincing comparative studies of dosing strategies. In these regulatory studies designed to find a dose to be compared with placebo or active drugs, a substantial proportion of patients failed dose titration with any product used to delivery transmucosal fentanyl. Moreover an unclear distinction between basal pain of mild-moderate intensity and BTP of moderatesevere intensity make the interpretation of data provided by these studies difficult ${ }^{10}$. In clinical practice, low doses of ROOs, started in an attempt to titrate the doses individually, are unlikely to produce any effect in patients receiving high doses of opioids for their background pain, and may result in unnecessary suffering. Dose titration may make the practical use of ROOs difficult in daily activity, particularly at home or in outpatients. Patients may be reluctant to try the dose and avoid using these drugs ${ }^{11,12}$, preferring, in the end, traditional oral dosing of morphine ${ }^{13}$.

A predictable dose could favour an easy prescription, resulting in better patient compliance. The principal problem concerns the risk of toxicity. However, the use of proportional doses has been shown to be safe and effective in a large number of patients, in open-label and controlled studies ${ }^{14-18}$. In the only existing study comparing titration strategy with the proportional approach, the latter was found to be more effective while the adverse effects were similar ${ }^{19}$.

Sublingual fentanyl (SLF) is a second generation ROO, formulated as a rapidly disintegrating tablet system containing a mixture of carrier particles coated with active drug particles and containing a mucoadhesive agent. The bioavailability of SLF is estimated to be about $70 \%{ }^{20}$, with an interindividual variability lower than that reported with oral transmucosal fentanyl ${ }^{21}$. Controlled studies have demonstrated the efficacy and safety of SLF for the management of $\mathrm{BTP}^{22,23}$. In the former study, higher doses were more effective, possibly in patients receiving higher doses of opioids used for background analgesia, and with similar profiles of adverse effects in comparison with lower doses, suggesting the use of proportional doses. The aim of this study was to prospectively assess the efficacy and safety of SLF in doses proportional to opioid doses used for background analgesia for the treatment of BTP of cancer patients admitted in an acute pain relief and supportive care unit. Age was also considered as a possible factor influencing the outcome. The secondary aim was to evaluate a possible early onset of SLF.

\section{Methods}

A sample of patients consecutively admitted to an acute palliative care unit in a period of 12 months, from May 2012 to April 2013, was surveyed. From this sample, patients who were receiving opioids in doses of oral morphine equivalents equal to or more than $60 \mathrm{mg}$ daily, and having well controlled background pain and presenting $\leq 3$ BTP episodes/day, were selected. Patients who were asked to participate were prescribed SLF in doses proportional to opioids used for background analgesia. According to consolidated local policy and previous published experience $^{14-19}$ to calculate the dose, for example, the minimal existing dose $100 \mu \mathrm{g}$ was given to patients receiving $60 \mathrm{mg}$ of oral morphine equivalents, $200 \mu \mathrm{g}$ was given to patients receiving $120 \mathrm{mg}$ of oral morphine equivalents, and so on. Informed consent and institutional approval were obtained.

Patients were treated according to a routine protocol. After establishing around the clock opioid medication, according to opioid titration process, achieving a stable analgesia, with mean pain intensity of $\leq 4 / 10$ (on a numerical scale of $0-10$ ), for two consecutive days, patients were instructed to call for administering SLF at the doses calculated when a superimposed episode of BTP occurred. For each episode of BTP, trained nurses recorded patients' assessed pain intensity (numerical scale $0-10$ ), and adverse effects measured on a scale from 0 to 3 (absent, mild, moderate, and severe), as well as adverse effects severe enough in intensity to require medical intervention. Recording was performed just before giving the SLF dose (TO), and 5, 10 and 15 minutes after (T5, T10, and T15, respectively). The administration of SLF was considered unsuccessful whenever further BTP medication was required in the subsequent 2 hours. To evaluate the efficacy of SFL, the number of episodes which were successfully treated using SLF in doses proportional to the basal opioid regimen, within 15 minutes, was assessed. Safety was assessed by measuring the changes in adverse effects intensity and the occurrence of adverse effects severe enough in intensity to require a medical intervention. Changes in pain 
Table 1. Characteristics of patients, number of episodes collected, mean doses of SLF ( $\mu \mathrm{g})$, oral morphine equivalents (mg/day), and changes in pain intensity at the different time intervals (see text). Standard deviation (SD) in brackets.

\begin{tabular}{lcccc}
\hline N $^{\circ}$ patients & All & Age $\leq 65$ yrs & Age $>65$ yrs & $p$ \\
\hline M/F & $41 / 29$ & $26 / 17$ & $15 / 12$ & 0.685 \\
Age & $61.7(11.5)$ & $54.6(8.1)$ & $73.1(5.2)$ & $/$ \\
Evaluable episodes & 173 & 111 & 62 & $/$ \\
Mean dose of SLF (SD) & $637(786)$ & $765(954)$ & $433(311)$ & $<0.0005$ \\
Oral morphine equivalents for background pain (SD) & $\mathbf{3 6 2}(\mathbf{3 2 0})$ & $\mathbf{4 2 0}(\mathbf{4 8 0})$ & $\mathbf{2 4 5}(\mathbf{2 1 0})$ & $<\mathbf{0 . 0 0 0 5}$ \\
TO Pain intensity & $7.0(1.1)$ & $7.1(1.1)$ & $6.7(0.9)$ & 0.016 \\
T5 Pain intensity & $6.0(1.1)$ & $6.1(1.2)$ & $5.9(0.9)$ & 0.152 \\
T10 Pain intensity & $4.9(1.4)$ & $4.9(1.4)$ & $4.8(1.4)$ & 0.535 \\
T15 Pain intensity & $3.6(1.6)$ & $3.6(1.5)$ & $3.5(1.7)$ & 0.218 \\
\hline
\end{tabular}

intensity were also assessed to evaluate possible earlier analgesic effects of SLF (5 and 10 minutes).

\section{Statistical analysis}

Frequency analysis was performed using Pearson's chisquared test and Fisher's exact test. One-way analysis of variance (ANOVA) and the Kruskal Wallis statistic test were used to compare the different parametric or nonparametric variables. For analysis, patients were divided according to age: $\leq 65$ years and $>65$ years. Data was analysed with SPSS Software 14.0 version (SPSS Inc., Chicago, IL, USA). All $p$-values were two sided, and $p$-values less than 0.05 were considered statistically significant. On the basis of previous similar studies ${ }^{14-19}$, a sample of 150 episodes was considered sufficient to determine the efficacy and safety of this approach.

\section{Results}

Seventy patients were administered SLF. The primary diagnosis was in a rank order: lung 24, uro-gynaecological 15, pancreas 10, colon 5, breast 5, others 11 . Characteristics of patients are reported in Table 1. The mean age was $61.7( \pm 11.5)$, and $27(38.6 \%)$ patients were over 65 years. Forty-one patients were males. A total of 173 episodes of BTP were recorded (mean 2.5 episodes/patient). In 19 events, documentation regarding changes in pain intensity was incomplete in the record sheet $(12,4,2,1$ events were incomplete at T0, T5, $\mathrm{T} 10$, and $\mathrm{T} 15$, respectively). Of the 154 evaluable episodes, 143 were successfully treated $(92 \%)$, without any further request. Mean doses of SLF are reported in Table 1. Fifty-one patients (72.8\%) received SLF doses $\geq 800 \mu \mathrm{g}$. When compared to younger adult patients, older patients received significantly lower doses of fentanyl buccal tablet (FBT) $(p<0.0005)$.

Pain intensity significantly decreased at T5, T10 and $\mathrm{T} 15(p=<0.0005)$. The percentage of patients with a pain reduction of more than $33 \%$ at $\mathrm{T} 5, \mathrm{~T} 10$, and $\mathrm{T} 15$, and the percentage of patients with a reduction in pain intensity of more than $50 \%$ at $\mathrm{T} 5, \mathrm{~T} 10$, and $\mathrm{T} 15$, are reported
Table 2. Number of BTP episodes with a decrease in $\mathrm{PI}>33 \%$ and $50 \%$, and changes of adverse effect intensity after administration of SLF.

\begin{tabular}{|c|c|c|c|c|}
\hline $\mathrm{N}^{\circ}$ patients & All & Age $\leq 65$ yrs & Age $>65$ yrs & $p$ \\
\hline \multicolumn{5}{|c|}{ Number of BTP episodes with a decrease in $\mathrm{PI}>33 \%$} \\
\hline At T5 & 11 & 8 & 3 & 0.748 \\
\hline At T10 & 79 & 48 & 31 & 0.428 \\
\hline At T30 & 137 & 85 & 52 & 0.329 \\
\hline \multicolumn{5}{|c|}{ Number of BTP episodes with a decrease in $\mathrm{PI}>50 \%$} \\
\hline At T5 & 1 & 1 & 0 & 1 \\
\hline At T10 & 21 & 15 & 6 & 0.628 \\
\hline At T15 & 114 & 69 & 45 & 0.184 \\
\hline \multicolumn{5}{|c|}{$\begin{array}{l}\text { Number of patients with adverse effects at baseline (T0) and after SLF } \\
(\mathrm{T} 5, \mathrm{~T} 10, \mathrm{~T} 15)\end{array}$} \\
\hline At To & 71 & 43 & 28 & 0.424 \\
\hline At T5 & 74 & 46 & 28 & 0.748 \\
\hline At T10 & 75 & 45 & 30 & 0.340 \\
\hline At T15 & 76 & 47 & 29 & 0.632 \\
\hline
\end{tabular}

$\mathrm{BT0}=$ breakthrough pain, $\mathrm{PI}=$ pain intensity, $\mathrm{SLF}=$ sublingual fentanyl

in Table 2. No differences in changes in pain intensity for gender $(p<0.9)$ or age $(p<0.85)$ were observed.

The main adverse effects, drowsiness and nausea, generally of low intensity, were already present, due to basal opioid treatment or disease. No significant changes in the number of patients reporting adverse effects of mildmoderate intensity were reported after SLF administration in comparison with baseline (numbers of patients and $p$ values are reported in Table 2). Finally, no adverse effects severe enough in intensity to require medical intervention were observed.

\section{Discussion}

The finding sof this study suggest that SLF, used in proportional doses to opioid basal regimen for the management of BTP, is as effective as safe, also considering that most patients received relatively high doses of SLF. This data confirms previous observations reported with other ROOs and intravenous morphine ${ }^{6}$, even in patients receiving high doses of opioids for background analgesia without adding risks of occurrence of adverse effects ${ }^{17}$, or in patients followed at home, which is the least protected setting ${ }^{24}$. 
These findings contradict general recommendations, which suggested that the dose of ROOs to be given for an episode for BTcP should be determined by individual titration ${ }^{7}$. There are several considerations which biased this statement, inherited in the literature, and largely examined in previous analyses ${ }^{11,12}$. During the titration phases reported in the literature, $10-30 \%$ of episodes treated may fail during dose titration, particularly in patients receiving high doses of opioids. As a consequence all these studies should be considered as enrichment trials, where the bad patients were excluded, and the responsive ones were compared with active substances (oral opioids) or placebo. The need of dose titration has never been specifically examined, and information gathered is just consequential to the study design aimed to demonstrate the superiority of ROOs over placebo or oral opioids, or to evaluate the safety and efficacy of ascending doses of ROOs. In fact, the titration period is an uncontrolled open phase ${ }^{12}$. The need for titration is only based on the observation that there is no correlation between the basal opioid regimen and the effective dose found after dose titration. The reasons for the lack of relationship between doses for BTP and basal opioid regimen have not been clearly explained, considering that the presence of tolerance should suggest a dose proportional to that used for background analgesia, according to consolidated experience with the use of oral opioids for BTP, although even this procedure is devoid of specific evidence.

From the practical point of view, dose titration may make the practical use of any ROO difficult in daily activity, particularly at home or in outpatient clinics. Considering the different presentation of each single episode, potentially titration should be performed for each event. Using different pieces of ROOs for treating each episode may be time consuming and may exceed the spontaneous duration for BTP which can spontaneously subside, as evidenced by the numerous successful placebo-treated patients reported in the literature ${ }^{11,12}$. As a consequence, patients should be prescribed more packages with different doses, unless using more pieces of lower doses. Finally, most patients may be reluctant to try the dose and avoid using these drugs, preferring, in the end, traditional oral dosing of morphine ${ }^{13}$.

It could be argued that a proportional dose without titration could expose patients to the risks of adverse effects. In practice, although dose titration may appear safer, at the end it requires doses similar to those resulting from proportional doses and may result in more failures, prolonging patients' suffering and reducing their compliance. A simulation of a calculation of doses of opioids used for background analgesia and those achieved after individual titration showed mean values of proportional doses very close to those found after titration ${ }^{10}$. In a 'real world' study reproducing a clinical scenario of patients receiving opioids for BTP, while the dose of oral opioids used as rescue medication was $18 \%$ of the around the clock opioid dose, for oral transmucosal fentanyl titrated to determine the effective dose, the rescue dose was about $35 \%$ of the around the clock dose ${ }^{25}$, suggesting that the titration process may provide even higher doses than those expected by using proportional doses to the basal regimen. Several observational studies on large sample of patients and controlled studies have shown that the use of proportional doses of ROOs is both effective and safe ${ }^{14-19}$. Recently, the titration method was compared with proportional doses of fentanyl buccal tablet (FBT), which has similar availability to that of SLF. In patients receiving proportional doses, efficacy was better in patients receiving doses of oral morphine equivalents of $>120 \mathrm{mg} /$ day, in comparison with the titration group, and the need of rescue doses was significantly more often reported in the titration group for the first episode of BTP. Importantly, this outcome was not associated with differences in adverse effects intensity between the two groups ${ }^{19}$. In other words, the use of doses proportional to the opioid basal regimen, easy calculable, could be more effective, without exposing patients to more adverse effects, as suggested by several practical experiences with intravenous morphine and ROOs, even at high doses and in elderly patients ${ }^{11,12}$. This is explained by the protective role of the level of tolerance, which is related with the doses of opioids used for the background pain. It has been shown that there is tolerance to adverse effects in patients chronically exposed to opioids, despite serum fentanyl levels as high as $6-8 \mathrm{ng} /$ $\mathrm{mL}^{26}$. Therefore, while titration is a mandatory process to optimize background analgesia in individuals, in a tolerant patient this process may loose priority, as patients have a known level of tolerance to opioids and a proportional dose may be predictably effective, without risks of adverse effects. The number of patients with possibly opioidinduced adverse effects did not change significantly after SLF administration. It is of interest that no difference was found in the intensity of adverse effects in comparison with episodes treated in patients receiving lower doses. Finally, no adverse effects severe enough in intensity to require medical intervention were observed. SLF was used even in high doses $(\geq 800 \mu \mathrm{g})$ in about $50 \%$ of patients and episodes, resulting safe other than being effective. Older patients received lower doses. This finding was expected and reflects the lower opioid doses used for background analgesia, as commonly reported in the literature ${ }^{27,28}$. These data suggest that SLF given in doses proportional to opioid basal regimen does not add to risk of overdosage, even in older patients or when used at high doses.

In this study it has also been shown that a decrease of $\geq 33 \%$ and $50 \%$ in pain intensity may be achievable in $51 \%$ and $13 \%$ of patients, respectively, within 10 minutes, while the figures were lower when assessing pain intensity at T5 (21\% and $1 \%$, respectively), suggesting an onset of clinical effect of 10 minutes in about $50 \%$ of patients, 
depending on which cut-off of pain intensity is used. This finding confirms pharmacokinetics studies in which a detectable plasma concentration of fentanyl occurred in 8-11 minutes ${ }^{21}$. A reduction of approximately two points or a reduction of approximately $30 \%$ of pain intensity has been reported to be a cut-off for clinically important difference ${ }^{29}$. Recently, in a study assessing meaningful pain intensity after BTP medication, the majority of patients requested a level of pain of 3.5 for adequate analgesia, which was half of the intensity of BTP ${ }^{30}$.

It is of interest that the availability of $300 \mu \mathrm{g}$ strength with SLF is an added value, when using proportional doses, as there is a minimal need to round the doses (for example in a patient receiving $180 \mathrm{mg} /$ day of oral morphine equivalents).

In a previous study of SLF performed in patients receiving an opioid regimen equivalent to $30-1000 \mathrm{mg} /$ day of oral morphine or $25-300 \mu \mathrm{g} / \mathrm{h}$ of transdermal fentanyl, $400 \mu \mathrm{g}$ of SLF was significantly more effective in reducing pain intensity and requirement for rescue analgesia than placebo, in comparison with $100 \mu \mathrm{g}$ and $200 \mu \mathrm{g}$ of SLF or placebo. Unfortunately the dose of around the clock opioid medication was not reported, and it was not possible to assess the relationship between fentanyl doses and basal opioid regimen. It is of interest that adverse effects were mild-moderate and did not increase with increasing SLF dose $^{22}$. In other words, higher doses of SLF were more effective, without adding to risk of adverse effects. In a traditional protocol where SLF was titrated to the successful dose, SLF was compared with placebo in patients receiving oral morphine equivalents of $60-1000 \mathrm{mg}$ or transdermal fentanyl in doses of $50-300 \mu \mathrm{g} / \mathrm{h}$. SLF provided significant improvements in pain intensity at 30 minutes and 60 minutes and from 10 minutes post-dose relative to placebo ${ }^{23}$. No raw data on doses of opioids were provided, so no analysis on possible correlations between basal opioid regimen and successful SLF dose was performed.

The principal limitation of this study is represented by the open-label non-comparative nature of this study. However, the aim was not to assess the efficacy in comparison with placebo, as this information already existed. This pilot study reproduces a typical clinical scenario where calculating the dose to be administered may provide easy prescription for patients while assuring efficacy and safety, as already observed with other fentanyl delivery systems. On the other hand, as mentioned before, titration phases in controlled studies were open.

\section{Conclusion}

SLF administered in doses proportional to basal opioid regimen for background pain seems to be effective, providing optimal analgesia in the majority of treated episodes of
BTP, and devoid of important adverse effects, reproducing previous observations reported with other ROOs.

\section{Transparency}

Declaration of funding

The study did not receive any economic support from pharmaceutical companies.

\section{Declaration of financial/other relationships}

S.M. has served on speakers bureaus for Takeda, Teva and Grunenthal and A.C. has served on speakers bureaus for Grunenthal, Janssen, Teva and Molteni Prostraken. G.P. declares no conflicts of interest.

CMRO peer reviewers may have received honoraria for their review work. The peer reviewers on this manuscript have disclosed that they have no relevant financial relationships.

\section{Acknowledgements}

The authors wish to thank the nursing staff of the unit for the support provided in collecting the data.

\section{References}

1. Portenoy RK, Hagen NA. Breakthrough pain: definition, prevalence and characteristics. Pain 1990;41:273-81

2. Davies A, Zeppetella G, Andersen S, et al. Multicentre European study of breakthrough cancer pain: characteristics and patient perceptions of current and potential management strategies. Eur J Pain 2011;15:756-63

3. Mercadante S, Valle A, Porzio G, et al. Relationship between background cancer pain, breakthrough pain, and analgesic treatment: a preliminary study for a better interpretation of epidemiological and clinical studies. Curr Med Res Opin 2013;29:667-71

4. Portenoy RK, Payne D, Jacobson P. Breakthrough pain: characteristics and impact in patients with cancer pain. Pain 1999;81:129-34

5. Hanks GW, De Conno F, Cherny N, et al. Morphine and alternative opioids in cancer pain: the EAPC recommendations. $\mathrm{Br} \mathrm{J}$ Cancer 2001;84:587-93

6. Mercadante S. Pharmacotherapy for breakthrough cancer pain. Drugs 2012;72:181-90

7. Davies A, Dickman A, Reid C, et al. The management of cancer-related breakthrough pain: recommendations of a task group of the Association of Palliative Medicine of Great Britain and Ireland. Eur J Pain 2009;13:331-8

8. Zeppetella G, Messina J, Xie F, et al. Consistent and clinically relevant effects with fentanyl buccal tablet in the treatment of patients receiving maintenance opioid therapy and experiencing cancer-related breakthrough pain. Pain Pract 2010;10:287-93

9. Kleeberg UR, Filbet M, Zeppetella G. Fentanyl buccal tablet for breakthrough cancer pain: why titrate? Pain Pract 2011;11:185-90

10. Mercadante $S$. Breakthrough pain: on the road again. Eur $J$ Pain 2009;13:329-30

11. Mercadante $\mathrm{S}$. Rapid onset opioids for breakthrough pain: titrating or not titration or not titrating, this is the question. Eur J Pain 2011;5(Suppl):443-8

12. Mercadante $\mathrm{S}$. The use of rapid onset opioids for breakthrough cancer pain: the challenge of its dosing. Crit Rev Oncol Hematol 2011;80:460-5

13. Davies A, Vriens J, Kennett A, et al. An observational study of oncology patients' utilization of breakthrough pain medication. J Pain Symptom Manage 2008;35:406-11 
14. Mercadante S, Villari $P$, Ferrera $P$, et al. Safety and effectiveness of intravenous morphine for episodic (breakthrough) pain using a fixed ratio with the oral daily morphine dose. J Pain Symptom Manage 2004;27: 352-9

15. Mercadante S, Intravaia G, Villari P, et al. Intravenous morphine for episodicbreakthrough pain in an acute palliative care unit: a confirmatory study. J Pain Symptom Manage 2008;35:307-13

16. Mercadante S, Villari P, Ferrera $P$, et al. The use of opioids for breakthrough pain in an acute palliative care unit by using doses proportional to opioid basal regimen. Clin J Pain 2010;26:306-9

17. Mercadante S, Ferrera P, Adile C, et al. The use of fentanyl buccal tablets in doses proportional to the doses of opioids used for background analgesia for the treatment of breakthrough cancer pain in highly tolerant cancer patients. J Pain Symptom Manage 2011;42:464-9

18. Mercadante $S$, Villari $P$, Ferrera $P$, et al. Transmucosal fentanyl vs intravenous morphine in doses proportional to basal opioid regimen for episodic-breakthrough pain. Br J Cancer 2007;96:1828-33

19. Mercadante S, Gatti A, Porzio G, et al. Dosing fentanyl buccal tablet for breakthrough cancer pain: dose titration versus proportional doses. Curr Med Res Opin 2013;29:93-7

20. Chwieduk C, McKeage K. Fentanyl sublingual in breakthrough pain in opioidtolerant adults with cancer. Drugs 2010;70:2281-8

21. Lennernas B, Hedner T, Holmberg M, et al. Pharmacokinetics and tolerability of different doses of fentanyl following sublingual administration of a rapidly dissolving tablet to cancer patients: a new approach to treatment of incident pain. Br J Clin Pharmacol 2004;52:249-53
22. Lennernas B, Frank-Lissbrant I, Lennernas $H$, et al. Sublingual administration of fentanyl to cancer patients is an effective treatment for breakthrough pain: results from a randomized phase II study. Palliat Med 2010;24:286-93

23. Rauck R, Tark M, Reyes $\mathrm{E}$, et al. Efficacy and long-term tolerability of sublingual fentany lorally disintegrating tablet in the treatment of breakthrough cancer pain. Curr Med Res Opin 2009;25:2877-85

24. The use of fentanyl buccal tablets for breakthrough pain by using doses proportional to opioid basal regimen in a home care setting. Support Care Cancer 2013:21:2335-9

25. Zeppetella G. Opioids for cancer breakthrough pain: a pilot study reporting patient assessment of time to meaningful pain relief. J Pain Symptom Manage 2008;35:563-7

26. Aronoff G, Brennan M, Pritchard D, et al. Evidence-based oral transmucosal fentanyl citrate (OTFC) dosing guidelines. Pain Med 2005;6:305-14

27. Viganò A, Bruera E, Suarez-Almazor M. Age, pain intensity, and opioid dose in patients with advanced cancer. Cancer 1998;83:1244-50

28. Mercadante S, Casuccio A, Pumo S, et al. Factors influencing the opioid response in advanced cancer patients with pain followed at home. The effects of age and gender. Support Care Cancer 2000;8:123-30

29. Farrar J, Polomano R, Berlin J, et al. A comparison of change in the 0-10 numeric rating scale to a pain relief scale and global medication performance scale in a short-term clinical trial of breakthrough pain intensity. Anesthesiology 2010;11:1464-72

30. Mercadante S, Adile A, Torta R, et al. Meaningful cut-off pain intensity for breakthrough pain changes in advanced cancer patients. Curr Med Res Opin 2013;29:93-7 